\title{
Association of hormonal state with cardiovascular risk evaluated with Globorisk in Mexican women
}

\author{
Eduardo González-Escudero, ${ }^{1}$ Lourdes Basurto-Acevedo, ${ }^{1}$ Susana Barrera-Hernández, ${ }^{1}$ \\ Alma Díaz-Martínez, ${ }^{1}$ Lucero Sánchez-Huerta, ${ }^{1}$ Oswaldo Hernández-González, ${ }^{1}$ \\ Gerardo Santiago-Jiménez, ${ }^{2}$ Víctor Manuel González-Quintana, ${ }^{1}$ Ana Karen Rodríguez-Luna ${ }^{2}$ and \\ Antonio González-Chávez ${ }^{3}$ \\ ${ }^{1}$ Instituto Mexicano del Seguro Social, Centro Médico Nacional, Specialty Hospital; ${ }^{2}$ Instituto Politécnico Nacional, Superior School of Medicine; \\ ${ }^{3}$ Secretaría de Salud, Hospital General de México, Department of Internal Medicine. Ciudad de México, Mexico
}

\begin{abstract}
Introduction: Cardiovascular disease is the main cause of mortality worldwide. In women, its incidence increases at the sixth decade of life, coinciding with postmenopause. Whether this effect is due to menopause-related hormonal changes is not known. Objective: To evaluate the differences in cardiovascular risk in pre- and postmenopausal women by means of the Globorisk risk scale, the triglyceride/high-density lipoproteins cholesterol (Tg/HDL-C) ratio and metabolic syndrome (MS) criteria. Method: Cross-sectional study that included 408 women from 40 to 60 years of age; anthropometric measurements and biochemical determinations were performed. The participants were classified as premenopausal and postmenopausal. Cardiovascular risk was assessed using the MS criteria, the Globorisk risk calculator and the Tg/HDL-C ratio. Results: Postmenopausal women showed a significant increase in waist circumference, total cholesterol and triglycerides in comparison with premenopausal women. Significant associations were found between hormonal state and Globorisk measured cardiovascular risk $(O R=2.50 ; 95 \% \mathrm{Cl}=1.67-3.74)$ and the $\mathrm{Tg} / \mathrm{HDL}-\mathrm{C}$ ratio $(\mathrm{OR}=1.66 ; 95 \% \mathrm{Cl}=1.09-2.52)$. Conclusion: Cardiovascular risk factors have a higher prevalence in postmenopause. The Globorisk scale and Tg/HDL-C ratio identify cardiovascular risk in postmenopausal women.
\end{abstract}

KEY WORDS: Menopause. Cardiovascular disease. Globorisk. Metabolic syndrome. Triglyceride/HDL-cholesterol ratio.

\section{Introduction}

The main cause of mortality in the world both in men and women is cardiovascular disease (CVD), ${ }^{1}$ which represents a serious problem for many Latin American countries; ${ }^{2}$ in Mexico, one woman dies every 15 minutes due to CVD. ${ }^{3}$ Morbidity and mortality from this cause is more common in men that in women until the sixth decade of life; ${ }^{4}$ in the female population, it appears 10 to 15 years later, a stage that coincides with postmenopause..$^{5}$ Building upon this observation, multiple studies have been carried out to identify whether hypoestrogenism during menopause directly or indirectly increases CVD when the prevalence of risk factors such as dyslipidemia, diabetes mellitus (DM), overweight and arterial hypertension (AH) is increased. ${ }^{6}$
There are more than 100 cardiovascular risk prediction models, including the metabolic syndrome (MS) criteria, ${ }^{7}$ the triglyceride/HDL-C index (Tg/HDL-C) ${ }^{8}$ and the Framingham scale; ${ }^{9}$ however, many of them have not been validated beyond the populations where they were developed..$^{10}$ In relation to this, the Globorisk risk scale was published, which is an adaptation of the Framingham tables for different countries, including Mexico. In this studio, Mexican women aged 40 to 84 years were observed to be at second place of CVD risk. ${ }^{11,12}$

Multiple cross-sectional and longitudinal studies have been carried out to identify if menopause is a cardiovascular risk (CVR) factor that is independent of age or body mass index (BMI), with results not yet conclusive. ${ }^{13}$
Correspondence:

Lourdes Basurto-Acevedo

E-mail: Ibasurtoa@yahoo.com
Date of reception: 11-01-2018

Date of acceptance: 02-08-2018

DOI: 10.24875/GMM.M19000204
Gac Med Mex. 2018;154:539-545

Contents available at PubMed www.gacetamedicademexico.com 
The present investigation was carried out with the purpose to identify the association between the menopausal status and some tools to assess CVR, such as Globorisk, the MS criteria and the Tg/HDL-C index, as well as to find out the differences in independent risk factors between premenopausal and postmenopausal women.

\section{Method}

Comparative, cross-sectional study in 710 women of 40 to 60 years of age and apparently healthy, who attended the Medical Research in Endocrine Diseases Unit of Siglo XXI National Medical Center of the Mexican Institute of Social Security in Mexico City. Women diagnosed with diabetes, liver failure, kidney disease, chronic infections, endocrinopathies, hematologic diseases or with a history of CVD were excluded. In addition, pregnant women, women with surgical menopause or who were on treatment with hormone replacement therapy were excluded. Finally, 408 women who met the selection criteria were selected.

This protocol was approved by the Mexican Institute of Social Security ethics committee. The participants were briefed on the study and signed the respective informed consent.

The participants were taken a medical history and underwent complete physical examination, where anthropometric measurements were recorded; height and weight were measured without shoes and with light clothing. Systolic and diastolic blood pressure was measured with an aneroid sphygmomanometer, in both arms, and the average of both measurements was used as final value. The participants were classified according to their BMl as having normal weight (BMI $\geq 18$ to 24.9), overweight (BMI $\geq 25$ to 29.9) or obesity (BMI $\geq 30$ ). Abdominal obesity was considered when waist circumference was $>80 \mathrm{~cm}$.

Antecubital venous blood samples were obtained at between 07:00 and 08:00 hours, after a fasting of more than 12 hours; the samples were collected in tubes without anticoagulant. The samples were centrifuged at $3500 \mathrm{rpm}$ for 15 minutes to separate aliquots, which were kept frozen at $-70^{\circ} \mathrm{C}$ until the performance of the tests. Glucose, high density lipoprotein cholesterol and triglyceride quantification was carried out in serum using the Ekem ${ }^{\circledast}$ semi-automated chemical analyzer (KontroLab). Low-density lipoprotein cholesterol (LDL-C) was calculated with Friedewald's LDL-C formula. ${ }^{14}$
Menopause was clinically defined by amenorrhea for more than 12 months and biochemically by follicle stimulating hormone (FSH) serum concentrations $>30 \mathrm{mU} / \mathrm{mL}$ and estradiol $<30 \mathrm{pg} / \mathrm{mL}$. The participants who were at perimenopause with irregular menstrual cycles or from had 3 to 12 months of amenorrhea were excluded from the study.

Cardiovascular risk was estimated by means of the Globorisk risk scale, ${ }^{11}$ which evaluates the following parameters: systolic blood pressure, total cholesterol, diabetes, smoking, age and gender. Based on this, five 10-year fatal cardiovascular risk categories were identified: < 3\%, 3-6 \%, 7-9 \%, 10-14\%, and $\geq 15 \%$. For the purposes of this study, two groups were considered: women with $<1 \%$ risk and a second group with $\geq 1 \%$ probability of having a fatal cardiovascular event at 10 years. ${ }^{15}$

MS was evaluated with the Harmonizing the Metabolic Syndrome consensus criteria, with the presence of at least 3 of the 5 following criteria: ${ }^{6}$

- Waist circumference $>80 \mathrm{~cm}$ for women.

- Triglycerides $(\mathrm{Tg}) \geq 150 \mathrm{mg} / \mathrm{dL}$ or intake of hypertriglyceridemia treatment.

- HDL cholesterol (HDL-C) $<50 \mathrm{mg} / \mathrm{dL}$.

- Systolic blood pressure $\geq 130 \mathrm{~mm} \mathrm{Hg}$ or diastolic $\geq 85 \mathrm{~mm} \mathrm{Hg}$, as well as a history of high blood pressure with or without treatment.

- Fasting glucose $\geq 100 \mathrm{mg} / \mathrm{dL}$.

The $\mathrm{Tg} / \mathrm{HDL}-\mathrm{C}$ index was obtained by dividing the serum triglyceride levels by HDL-C concentrations. High risk was considered when the index was $\geq 3.0$ and lower risk with an index $<3.0 .^{16}$

In order to identify the distribution of variables, normality tests such as mental methods, bias, kurtosis and the Kolmogorov-Smirnov test were used. Parametric variables are represented with the mean and standard deviation (SD), whereas for nonparametric variables, medians and interquartile ranges (IQR, 25$75 \%$ ) were used.

Hormonal status, MS and each of its components and the $\mathrm{Tg} / \mathrm{HDL}-\mathrm{C}$ index were considered as dichotomous qualitative variables. The number of SM components and Globorisk scale-measured cardiovascular risk were considered as ordinal variables, whereas the type of dyslipidemia was regarded as a polytomic variable. The remaining variables were considered to be quantitative.

To identify the mean differences between the premenopausal and postmenopausal women groups, Student's t-test was used for independent samples, whereas for nonparametric variables, Mann-Whitney's 
U-test was used. For qualitative variables, the chisquare test was used. To identify correlations, Pearson or Spearman's correlation coefficients were used according to the variable distribution. Risk was calculated with the odds ratio (OR) and $95 \%$ confidence intervals (Cl); for adjustment by age, the beta exponent was used. A $p$-value $<0.05$ was considered to be statistically significant. The statistical analysis was carried out with the SPSS program, version 21.

\section{Results}

Of 710 women, 408 who met the selection criteria were included; of them, $178(43.6 \%)$ were premenopausal women and 230 (56.4\%) were at postmenopausal stage. Differences were observed between pre- and postmenopausal women in age (46.0 \pm 3.3 years versus $52.5 \pm 3.9$ years, $p=0.001$ ), waist circumference $(88.7 \pm 11.8 \mathrm{~cm}$ versus $91.3 \pm 11.6 \mathrm{~cm}$, $\mathrm{p}=0.025)$, total cholesterol $(230.2 \pm 45.8 \mathrm{mg} / \mathrm{dL}$ versus $241.1 \pm 51.8 \mathrm{mg} / \mathrm{dL}, p=0.027$ ), triglycerides (121 mg/dL, IQR = 93-165 versus $138 \mathrm{mg} / \mathrm{dL}, 108-$ $192 \mathrm{mg} / \mathrm{dL}, \quad p=0.01$ ); and in $\mathrm{Tg} / \mathrm{HDL}-\mathrm{C}$ index (2.62 $\mathrm{mg} / \mathrm{dL}, I Q R=1.76-3.5$ versus $2.89 \mathrm{mg} / \mathrm{dL}$, 1.88-4.47, $p=0.039$ ). General characteristics of the population are shown in Table 1. In addition, a prevalence of overweight and obesity of $78.3 \%$ was found in both groups, $77.4 \%$ in premenopausal and $79 \%$ in postmenopausal women (Table 2).

Postmenopausal women showed a higher prevalence of MS and Tg/HDL-C index elevation; however, the differences were not significant (Table 2). In contrast, risk distribution according to the Globorisk scale was different between pre- and postmenopausal women (Table 2). Based on the cutoff point of $3 \%$, which defines very low cardiovascular risk $(<3 \%)$ or low cardiovascular risk (>3\%), the prevalence of this risk in the group of premenopausal women was $1.2 \%$, and in the postmenopausal group, $2.3 \%$. Therefore, cardiovascular risk based on the Globorisk scale was dichotomized as $<1 \%$ or $\geq 1 \%$. Postmenopausal women showed a higher proportion in estimated risk for fatal cardiovascular disease $(>1 \%)$ in comparison with premenopausal women, as shown in figure 1. Similarly, significant differences were found in the Tg/HDL-C index (Fig. 1).

The risk for hyperglycemia, hypertension and hypertriglyceridemia was observed to be higher in postmenopause (Table 3). In addition, the postmenopausal stage was associated with higher risk of cardiovascular events as assessed by the Globorisk scale, with an $\mathrm{OR}=2.50(95 \% \mathrm{Cl}=1.67-3.74)$, and with the $\mathrm{Tg} /$ HDL-C index, with an OR $=1.66(95 \% \mathrm{Cl}=1.09-2.52)$. However, when adjustment was made for age, these associations disappeared, except in the subgroup of women of 45 to 49 years of age, where the association was with the $\mathrm{Tg} / \mathrm{HDL}-\mathrm{C}$ index, with an $\mathrm{OR}=$ 2.35 (1.02-5.39) (Table 3).

\section{Discussion}

This study demonstrated that, during the postmenopausal stage, women show a CVR increase as assessed by the Globorisk scale and the Tg/HDL-C index. Identification of risk during the postmenopausal stage can be useful in CVD prevention. During this stage, women are known to have more visceral adipose tissue and an altered lipid profile in comparison with premenopausal women, which can contribute to a CVR increase during postmenopause. ${ }^{17}$

In contrast, no significant differences were found in this study in the prevalence of MS between pre- and postmenopausal women. MS increases the risk of mortality to a greater extent in women versus men, although controversy remains on whether menopause is an age-independent risk factor for CVD. ${ }^{18}$

This study identified a prevalence of MS of $42.9 \%$ in postmenopausal women, similar to that reported in other countries where prevalence ranges from 26.1 to $54.6 \%$, including a study in Mexico where a prevalence of $31 \%$ was reported..$^{19}$ The differences in prevalence are partly explained by the criteria that are used for diagnosis. In a previous study in women, MS prevalence in postmenopause was $44.4 \%$ with the ATP-III-NCEP criteria and $61.5 \%$ with the International Diabetes Federation criteria. ${ }^{20}$

The Tg/HDL-C index has been associated with insulin resistance, and this in turn with CVD. ${ }^{21}$ However, there are differences regarding the cutoff point in each population to identify insulin resistance. In this work, a cutoff point of 3.0 was used, similar to a previous study in a Mexican population, where high correlation with insulin resistance was demonstrated. ${ }^{16}$ Similarly, an increase in the Tg/HDL-C index in postmenopausal women has been observed in other works, and this index has been shown to be a useful MS predictor. ${ }^{22}$ Several investigations have been carried out with the purpose to identify whether menopause increases CVR, and for that purpose, risk calculators have been used, including SCORE. ${ }^{23} \mathrm{~A}$ previous work identified that, with this scale, there is a significant risk increase in postmenopausal women. ${ }^{23}$ 
Table 1. General characteristics of the population according to pre- and postmenopausal hormonal status

\begin{tabular}{|c|c|c|c|c|c|c|c|}
\hline \multirow[t]{2}{*}{ Variable } & \multicolumn{2}{|c|}{ Total $(n=408)$} & \multicolumn{2}{|c|}{ Premenopause $(n=178)$} & \multicolumn{2}{|c|}{ Postmenopause $(n=230)$} & \multirow[t]{2}{*}{$p$} \\
\hline & \multicolumn{2}{|c|}{ Mean \pm SD } & \multicolumn{2}{|c|}{ Mean \pm SD } & \multicolumn{2}{|c|}{ Mean \pm SD } & \\
\hline Age (years) & \multicolumn{2}{|c|}{$49.6 \pm 4.8$} & \multicolumn{2}{|c|}{$46.0 \pm 3.3$} & \multicolumn{2}{|c|}{$52.5 \pm 3.9$} & 0.001 \\
\hline Weight (kg) & \multicolumn{2}{|c|}{$68.7 \pm 12.3$} & \multicolumn{2}{|c|}{$68.9 \pm 13$} & \multicolumn{2}{|c|}{$68.5 \pm 11.7$} & 0.74 \\
\hline Height (m) & \multicolumn{2}{|c|}{$1.54 \pm 0.05$} & \multicolumn{2}{|c|}{$1.55 \pm 0.05$} & \multicolumn{2}{|c|}{$1.53 \pm 0.07$} & 0.290 \\
\hline Waist circumference (cm) & \multicolumn{2}{|c|}{$90.2 \pm 11.7$} & \multicolumn{2}{|c|}{$88.7 \pm 11.8$} & \multicolumn{2}{|c|}{$91.3 \pm 11.6$} & 0.025 \\
\hline BMI & \multicolumn{2}{|c|}{$28.7 \pm 5.0$} & \multicolumn{2}{|c|}{$28.2 \pm 5.3$} & \multicolumn{2}{|c|}{$28.8 \pm 4.7$} & 0.88 \\
\hline Systolic BP (mm Hg) & \multicolumn{2}{|c|}{$113.6 \pm 14.3$} & \multicolumn{2}{|c|}{$112.1 \pm 14$} & \multicolumn{2}{|c|}{$114.7 \pm 14.6$} & 0.069 \\
\hline Diastolic BP (mm Hg) & \multicolumn{2}{|c|}{$76.9 \pm 9.2$} & \multicolumn{2}{|c|}{$76.4 \pm 9.8$} & \multicolumn{2}{|c|}{$77.3 \pm 8.8$} & 0.339 \\
\hline Glucose (mg/dL) & \multicolumn{2}{|c|}{$88.5 \pm 22.2$} & \multicolumn{2}{|c|}{$87.2 \pm 20$} & \multicolumn{2}{|c|}{$89.5 \pm 23.8$} & 0.304 \\
\hline Triglycerides (mg/dL) & \multicolumn{2}{|c|}{$130(101-189)$} & 12 & 165) & & -192) & 0.010 \\
\hline Total cholesterol (mg/dL) & 236. & 19.5 & 230 & 45.8 & & 51.8 & 0.027 \\
\hline HDL-C (mg/dL) & 51. & & & & & 15.8 & 0.822 \\
\hline LDL-C (mg/dL) & 154 & & & 47.5 & & 47.5 & 0.071 \\
\hline & Mediana & IQR & Mediana & IQR & Mediana & IQR & \\
\hline Tg/HDL-C index & 2.7 & $1.8-4.1$ & 2.60 & $1.76-3.50$ & 2.89 & $1.88-4.47$ & 0.039 \\
\hline Estradiol (pg/mL) & 48.2 & $20.7-101$ & 77.5 & $49-140$ & 19.7 & $9.3-40.2$ & 0.001 \\
\hline MS components & & & & & & & \\
\hline 0 & & & & & & & 0.15 \\
\hline 1 & & & & & & & \\
\hline 2 & & & & & & & \\
\hline 3 & & & & & & & \\
\hline 4 & & & & & & & \\
\hline 5 & & & & & & & \\
\hline
\end{tabular}

$\mathrm{IQR}=25-75 \%$ interquartile range, $\mathrm{BMI}=$ body mass index, $\mathrm{BP}=$ blood pressure, $\mathrm{MS}=$ metabolic syndrome.

In the described study, a large proportion of participants were found to have low CVD risk $(<3 \%)$ and no women were found with high or very high risk, since diabetic patients were excluded. ${ }^{11}$ Conversely, in Globorisk original report, a prevalence of fatal risk was identified in $69 \%$ of Mexican women; it should be noted that, in this work, the women's age range was from 40 to 80 years of age and population with diabetes was included. ${ }^{11}$

In Globorisk second report, where cardiovascular risk was assessed based on biochemical and clinical factors and that was recalibrated for 182 countries, cardiovascular risk was observed to be increasing in developing countries in comparison with developed countries. ${ }^{12}$ The calibrated evaluation for Mexico, with a 15 -year projection, was $11 \%$ for women aged 40 to 84 years. ${ }^{11}$

Recalibration of the scale to each country and to each population would allow for the Globorisk prediction model to be improved and this way it could serve to establish CVD prevention strategies.

The prevalence of risk factors varies according to the population, genetic profile, age, dietary habits, lifestyle, physical activity, gender and hormonal status, and thus we consider this study to be a contribution for CVR identification in the female population. To the best of our knowledge, this is the first prospective study conducted using the Globorisk cardiovascular risk scale in women aged 40 to 60 years, since in this group of patients there is an increased risk for CVD. 
Table 2. Distribution of main risk factors in pre- and postmenopausal women

\begin{tabular}{|c|c|c|c|c|}
\hline \multirow[t]{2}{*}{ Variable } & \multirow{2}{*}{$\frac{\text { Total }(n=408)}{\%}$} & \multirow{2}{*}{$\frac{\text { Premenopause }(n=178)}{\%}$} & \multirow{2}{*}{$\frac{\text { Postmenopause }(\mathrm{n}=\mathbf{2 3 0})}{\%}$} & \multirow[t]{2}{*}{$p$} \\
\hline & & & & \\
\hline \multicolumn{5}{|c|}{ Abdominal obesity } \\
\hline Without & 16.8 & 20.0 & 14.4 & 0.13 \\
\hline With & 83.2 & 80.0 & 85.2 & \\
\hline \multicolumn{5}{|l|}{ BMl } \\
\hline Normal & 21.7 & 22.6 & 21.0 & 0.22 \\
\hline Overweight & 41.4 & 43.5 & 39.7 & \\
\hline Obesity & 36.9 & 33.9 & 39.3 & \\
\hline \multicolumn{5}{|c|}{ Arterial hypertension } \\
\hline Without & 66.1 & 68. & 64.4 & 0.49 \\
\hline With & 33.9 & 31.4 & 35.6 & \\
\hline \multicolumn{5}{|l|}{ Smoking habit } \\
\hline Without & 68.4 & 69.1 & 67.8 & 0.78 \\
\hline With & 31.6 & 30.9 & 32.2 & \\
\hline \multicolumn{5}{|l|}{ Hyperglycemia } \\
\hline Without & 82.8 & 83.1 & 82.6 & 0.99 \\
\hline With & 17.2 & 16.9 & 17.4 & \\
\hline \multicolumn{5}{|c|}{ Hypertriglyceridemia } \\
\hline Without & 59.7 & 65.9 & 51.7 & 0.44 \\
\hline With & 40.3 & 34.1 & 42.2 & \\
\hline \multicolumn{5}{|c|}{ Decreased HDL-C } \\
\hline Without & 49.3 & 50.3 & 48.6 & 0.82 \\
\hline With & 50.7 & 49.7 & 51.4 & \\
\hline \multicolumn{5}{|c|}{ Metabolic syndrome } \\
\hline Without & 59.3 & 62.7 & 57.1 & 0.31 \\
\hline With & 40.7 & 37.3 & 42.9 & \\
\hline \multicolumn{5}{|c|}{ Elevated Tg/HDL-C index } \\
\hline Without & 57.1 & 64.2 & 51.9 & 0.24 \\
\hline With & 42.9 & 35. & 48.1 & \\
\hline \multicolumn{5}{|l|}{ Globorisk } \\
\hline$<1$ & 52.0 & 64.4 & 42.2 & 0.05 \\
\hline 1 & 40.7 & 32.1 & 47.7 & \\
\hline 2 & 5.4 & 2.3 & 7.8 & \\
\hline 3 & 1.7 & 0.6 & 2.3 & \\
\hline 4 & 0.2 & 0.6 & 0 & \\
\hline
\end{tabular}

Table 3. Association of risk factors with postmenopausal status

\begin{tabular}{lcccr}
\hline Variable & $\mathbf{n}^{*}$ & $\%$ & OR (95\% Cl) & OR (95 \% Cl) \\
\hline Abdominal obesity risk & $198 / 408$ & 85.6 & $1.48(0.88-2.50)$ & $1.27(0.63-2.54)$ \\
Hypertension risk & $69 / 408$ & 24.8 & $3.12(1.74-5.38)$ & $0.44(1.43-1.73)$ \\
Hyperglycemia risk & $40 / 408$ & 17.4 & $1.03(0.61-1.74)$ & $1.19(0.56-2.55)$ \\
Hypertriglyceridemia risk & $103 / 404$ & 44.9 & $1.57(1.03-2.39)$ & $0.92(0.52-1.65)$ \\
HDL-C risk & $120 / 404$ & 51.4 & $1.07(0.71-1.61)$ & $1.52(0.85-2.70)$ \\
Metabolic syndrome & $106 / 404$ & 61.1 & $1.26(0.80-1.99)$ & $0.97(0.52-1.81)$ \\
Tg/HDL-C index & $112 / 404$ & 48.1 & $1.66(1.09-2.52)$ & $1.36(0.76-2.43)$ \\
Tg/HDL-C (45-49 years) & $19 / 118$ & 48.7 & $2.33(1.04-5.11)$ & $2.35(1.02-5.39)$ \\
Globorisk & $133 / 408$ & 57.8 & $2.50(1.67-3.74)$ & $1.06(0.61-1.84)$ \\
\hline
\end{tabular}

${ }^{*}$ Postmenopausal population. ${ }^{* *}$ Age-adjusted. $\mathrm{OR}=$ odds ratio, $\mathrm{Tg}=$ triglycerides. 


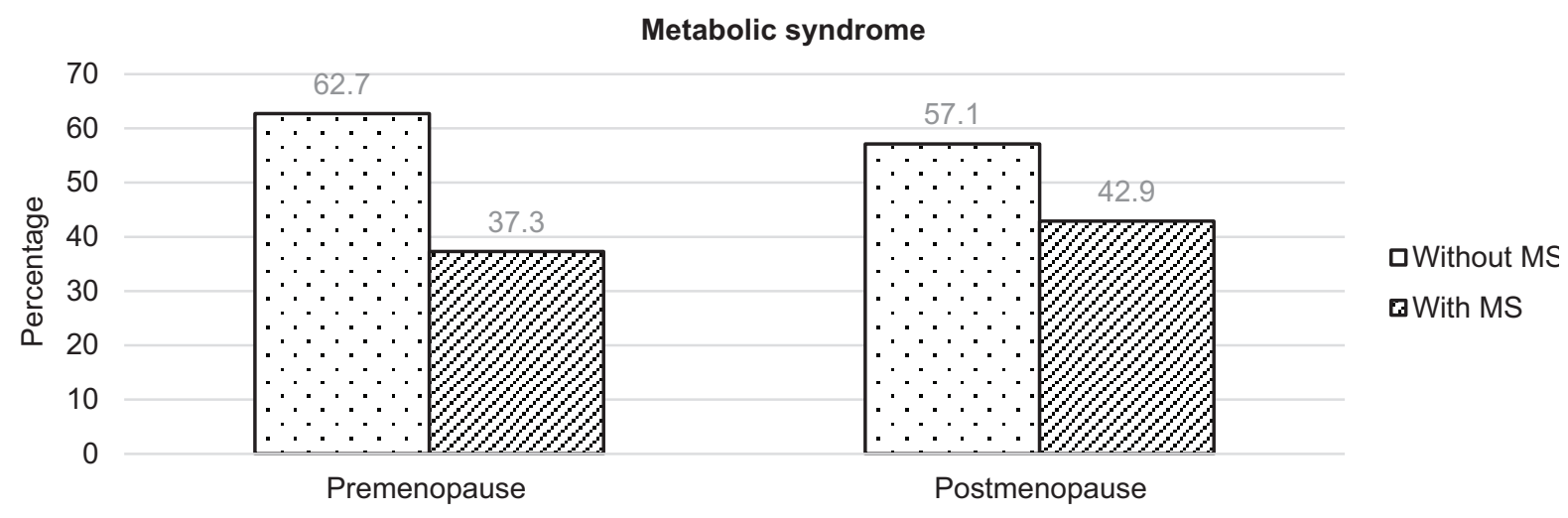

Risk assessed by Tg-HDL-C index

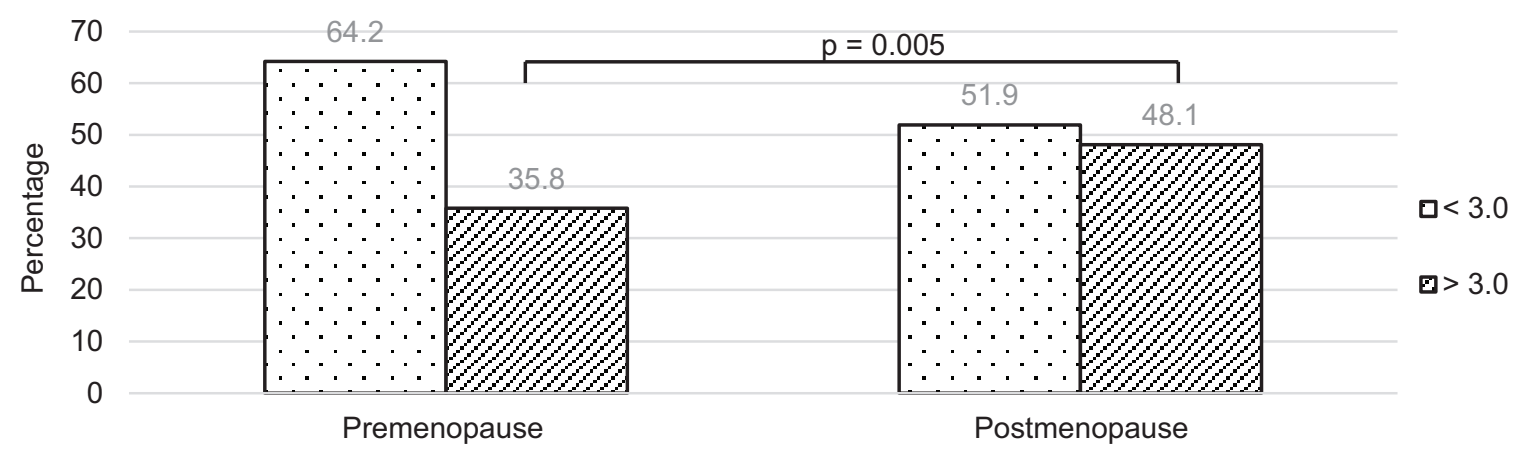

Risk assessed by Globorisk

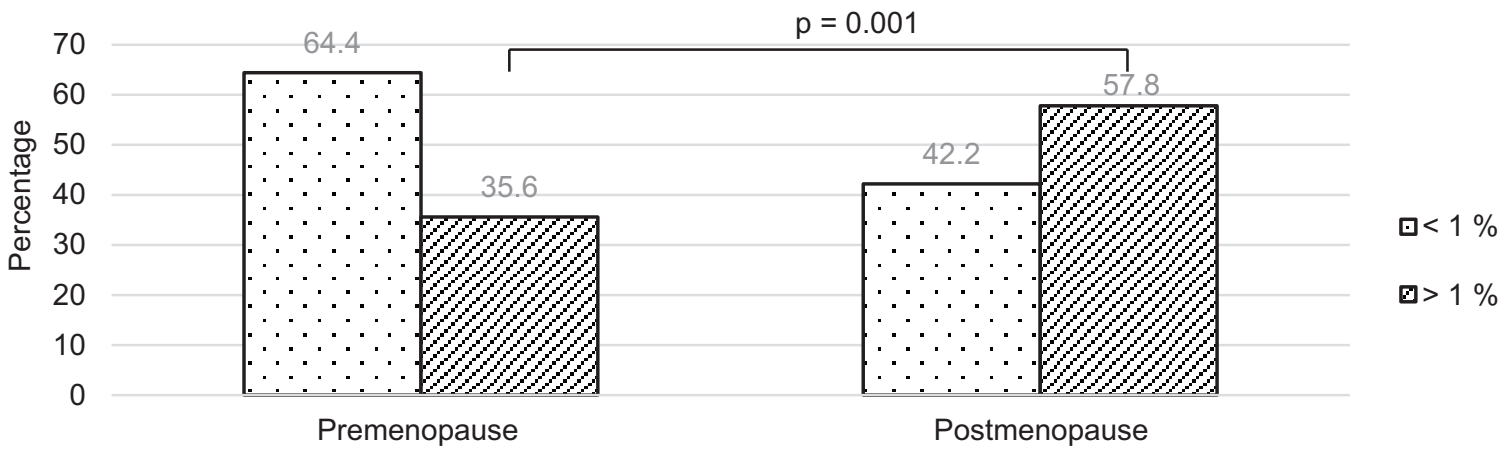

Figure 1. Differences in metabolic syndrome frequency, triglyceride/HDL cholesterol index (Tg/HDL-C) increase and Globorisk scale-assessed cardiovascular risk in pre- and postmenopausal women.

One limitation of the investigation lies in that the assessment was aimed at non-diabetic population and, therefore, the results show lower cardiovascular risk than that which can be observed in the general population. However, based on the results, it should be insisted on that, during the climacterium, cardiovascular disease prevention should be continued in apparently healthy women.

In summary, the data showed that cardiovascular risk factors have a higher prevalence during postmenopause, even in non- diabetic population. The Globorisk scale and the Tg/HDL-C index allow for the increase in cardiovascular risk to be identified in postmenopausal woman.

\section{Acknowledgements}

This study was financed by the Fund for Health Research of the Mexican Institute of Social Security.

\section{References}

1. GBD 2013 Mortality and Causes of Death Collaborators. Global, regional, and national age-sex specific all-cause and cause-specific mortality for 240 causes of death, 1990-2013: a systematic analysis for the Global Burden of Disease Study 2013. Lancet. 2015;385:117-71. 
2. Global status report on noncommunicable diseases 2010. Ginebra: World Health Organization; 2011.

3. Instituto Nacional de Estadística y Geografía. Causas de mortalidad. Defunciones generales de mujeres por principales causas de mortalidad, 2013. México: Instituto Nacional de Estadística y Geografía; 2015.

4. Artaud-Wild SM, Connor SL, Sexton G, Connor WE. Differences in coronary mortality can be explained by differences in cholesterol and saturated fat intakes in 40 countries but not in France and Finland. A paradox. Circulation. 1993;88:2771-2779.

5. Rossouw JE. Hormones, genetic factors, and gender differences in cardiovascular disease. Cardiovasc Res. 2002;53:550-557.

6. Agrinier N, Cournot M, Dallongeville J, Arveile D, Ducimetiere P, Ruidavets JB, et al. Menopause and modifiable coronary heart disease risk factors: a population based study. Maturitas. 2010;65:237-243.

7. Alberti KG, Eckel RH, Grundy SM, Zimmet PZ, Cleeman JI, Donato KA, et al. Harmonizing the metabolic syndrome: a joint interim statement of the International Diabetes Federation Task Force on Epidemiology and Prevention; National Heart, Lung, and Blood Institute; American Heart Association; World Heart Federation; International Atherosclerosis Society; and International Association for the Study of Obesity. Circulation. 2009;120:1640-1645.

8. Salazar MR, Carbajal HA, Espeche WG, Aizpurua M, Leiva-Sisnieguez CE Leiva-Sisnieguez BC, et al. Use of the plasma triglyceride/high-density lipoprotein cholesterol ratio to identify cardiovascular disease in hypertensive subjects. J Am Soc Hypertens. 2014;8:724-731.

9. D'Agostino RB, Vasan RS, Pencina MJ, Wolf PA, Cobain M, Massaro JM, et al. General cardiovascular risk profile for use in primary care: the Framingham Heart Study. Circulation. 2008;117:743-753.

10. Matheny M, McPheeters ML, Glasser A, Mercado N, Weaver RB, Jerome RN, et al. Systematic review of cardiovascular disease risk assessment tools. Rockville (MD): Agency for Healthcare Research and Quality (US); 2011.

11. Hajifathalian K, Ueda $P$, Lu $Y$, Woodward M, Ahmadvand A, Aguilar-Salinas CA, et al. A novel risk score to predict cardiovascular disease risk in national populations (Globorisk): a pooled analysis of prospective cohorts and health examination surveys. Lancet Diabetes Endocrinol. 2015;3:339-355.

12. Ueda P, Woodward M, Lu Y, Hajifathalian K, Al-Wotayan R, Aguilar-Salinas CA, et al. Laboratory-based and office-based risk scores and charts to predict 10-year risk of cardiovascular disease in 182 countries: pooled analysis of prospective cohorts and health surveys. Lancet Diabetes Endocrinol. 2017;5:196-213.

13. Del Principe D, Ruggieri A, Pietraforte D, Villani A, Vitale C, Straface E, et al. The relevance of estrogen/estrogen receptor system on the gender difference in cardiovascular risk. Int J Cardiol. 2015;187:291-298.

14. Friedewald WT, Levy RI, Fredrickson DS. Estimation of the concentration of low-density lipoprotein cholesterol in plasma, without use of the preparative ultracentrifuge. Clin Chem. 1972;18:499-502.

15. Secretaría de Salud. Diagnóstico y tratamiento de dislipidemias (hipercolesterolemia) en el adulto. México: México: Secretaría de Salud; 2016.

16. González-Chávez A, Simental-Mendía LE, Elizondo-Argueta S. Elevated triglycerides/HDL-cholesterol ratio associated with insulin resistance. Cir Cir. 2011;79:126-131.

17. Díaz-Martínez AG, Basurto-Acevedo L, Vega-García S, Saucedo-García R, Hernández-Valencia M, Zárate-Treviño A, et al. Evaluación del tejido adiposo visceral y de la concentración de lípidos en mujeres pre y posmenopáusicas. Ginecol Obstet Mex. 2016;84:294-300.

18. Donato GB, Fuchs SC, Oppermann K, Bastos C, Spritzer PM. Association between menopause status and central adiposity measured at different cutoffs of waist circumference and waist-to-hip ratio. Menopause. 2006;13:280-285.

19. Rojas JS, Lopera VJS, Cardona VJ, Vargas GN, Hormaza MP. Síndrome metabólico en la menopausia, conceptos clave. Rev Chil Obstet Ginecol. 2014;79:121-128.

20. Figueiredo-Neto JA, Figueredo ED, Barbosa JB, Barbosa-Fde F, Costa GR, Nina VJ, et al. Metabolic syndrome and menopause: cross-sectional study in gynecology clinic. Arq Bras Cardiol. 2010;3:339-345.

21. McLaughlin T, Reaven G, Abbasi F, Lamendola C, Saad M, Waters D, et al. Is there a simple way to identify insulin-resistant individuals at increased risk of cardiovascular disease? Am J Cardiol. 2005;96:399-404.

22. Arthur FK, Adu-Frimpong M, Osei-Yeboah J, Mensah FO, Owusu, L. Prediction of metabolic syndrome among postmenopausal Ghanaian women using obesity and atherogenic markers. Lipids Health Dis. 2012;11:101.

23. Piskorz A, Brzostek T. Comparison of SCORE-predicted risk of death due to cardiovascular events in women before and after menopause. Prz Menopauzalny. 2015;14:168-172. 